\title{
BMJ Open MAGNETO cardiography parameters to predict future Sudden Cardiac Death (MAGNETO-SCD) or ventricular events from implantable cardioverter defibrillators: study protocol, design and rationale
}

Thomas Lachlan, ${ }^{1}$ Hejie He, ${ }^{1}$ Kavi Sharma, ${ }^{1}$ Jamal Nasir Khan, ${ }^{1,2}$ Kim Rajappan, ${ }^{3}$ Adrian Morley-Davies, ${ }^{4}$ Ashish Patwala, ${ }^{4}$ Harpal Randeva, ${ }^{1,2}$ Faizel Osman (D) ${ }^{1,2}$

To cite: Lachlan $\mathrm{T}$, He $\mathrm{H}$ Sharma K, et al. MAGNETO cardiography parameters to predict future Sudden Cardiac Death (MAGNETOSCD) or ventricular events from implantable cardioverter defibrillators: study protocol, design and rationale. BMJ Open 2020;10:e038804. doi:10.1136/ bmjopen-2020-038804

- Prepublication history and additional materials for this paper is available online. To view these files, please visit the journal online (http://dx.doi. org/10.1136/bmjopen-2020038804).

Received 24 March 2020 Revised 23 August 2020 Accepted 06 September 2020

Check for updates

(C) Author(s) (or their employer(s)) 2021. Re-use permitted under CC BY-NC. No commercial re-use. See rights and permissions. Published by BMJ.

For numbered affiliations see end of article.

Correspondence to Professor Faizel 0sman; Faizel.osman@warwick.ac.uk

\section{ABSTRACT}

Introduction Predicting sudden cardiac death (SCD) is challenging as current risk predictors have significant limitations. Evaluating magnetocardiogram (MCG) parameters could be of great value and we plan to assess the capability of a new mobile unshielded MCG device in predicting SCD and ventricular arrhythmias (VA) in patients undergoing implantable cardioverter defibrillator (ICD) implantation.

Methods and analysis A prospective multicentre (University Hospitals Coventry and Warwickshire (UHCW) National Health Service (NHS) Trust/University Hospital North Midlands NHS Trust, UK) observational study evaluating the VitalScan MCG (Creavo Medical Technologies, UK) to predict future VA risk; 270 patients meeting criteria for primary or secondary prevention ICDs (ischaemic or non-ischaemic aetiology) are being recruited. The first patient was recruited September 2019 and the study will be completed at final participant followup. The primary endpoint is appropriate ICD therapy for VA, secondary endpoint is SCD. Previous trials using MCG identified late $\mathrm{QRS}$ signals/QRS fragmentation as potential indicators of SCD in small samples using large shielded expensive MCG devices that were difficult to use clinically. It is hoped the MAGNETO-SCD trial will show this new MCG device can provide real world risk stratification for SCD/NA risk. The trial has recruited 25 patients (13 with secondary prevention indication) from a single site (UHCW) with recruitment starting at the second site in March 2020. Ethics and dissemination Research Ethics Committee, Yorkshire and Humber Sheffield Research Ethics Committee UK (Ref: 19/YH/0143) and Health Research Authority (IRAS reference 254466, EDGE ID: 123146) approval received on 17/07/2019. The Medicines and Healthcare products Regulatory Agency approval received $11 / 07 / 2019$. Results will be disseminated via a peerreviewed publication and presentation at international conferences.

Trial registration numbers ClinicalTrials.gov Registry (NCT04352816) and EU Clinical Trials Registry (EudraCT2019-002994-78).
Strengths and limitations of this study

- This study is the first to explore the use of magnetocardiography (MCG) using a new device to determine its utility as a clinical risk predictor of ventricular arrhythmias and sudden cardiac death (SCD).

- It is a multicentre trial that will recruit patients with both primary and secondary prevention indications for an implantable cardioverter defibrillator and of any underlying aetiology allowing a truly unselected cohort to be evaluated.

- The new MCG device is portable and far less expensive compared with traditional older MCG technology.

- MCG waveforms may be dynamic just like surface 12-lead ECGs in patients with an arrhythmogenic substrate and therefore a single MCG may be a limitation.

- The study will not identify patients currently deemed low risk of SCD who may in fact be high risk and further studies of MCG in the general population will be needed.

\section{INTRODUCTION}

Sudden cardiac death (SCD) is responsible for over 4 million deaths globally per year with the predominant mechanism being cardiac ventricular arrhythmias (VAs). ${ }^{12}$ The only effective therapy, at present, for patients in cardiac arrest with these VA is defibrillation. The development and success of the implantable cardioverter defibrillator (ICD) has led to this being a first-line treatment for patients identified as being at high risk of SCD. ${ }^{3-6}$ However, SCD remains a significant cause of death with the majority occurring in groups deemed to be low risk. Conversely, a significant number of patients receive an ICD which 
is never used. This incurs a significant cost implication for healthcare services worldwide and more importantly exposes patients to potentially unnecessary device-related morbidity such as inappropriate shocks or device infection. Due to these demonstrable limitations in SCD risk assessment, there is a clear need for clinical research in this area. The genesis of VA has been described as a 'perfect storm' of vulnerable substrate and multiple transient factors that participate in triggering the VA event. ${ }^{2}$ Identifying this vulnerable substrate is considered a key to the puzzle of risk prediction for SCD but remains elusive to date.

Heterogeneous electrical conduction within the myocardium is considered a key factor for the development of VA. Myocardial scar and ischaemia have been identified as causing delay in electrical conduction, creating a disordered wave front that allows for the genesis of re-entrant circuits within the ventricular myocardium. ${ }^{7}$ Any ideal tool for identifying this heterogeneous conduction would provide sensitive and specific measures for SCD risk, while being inexpensive, non-invasive, low risk and well tolerated by patients. Not surprisingly, surface ECG has been extensively studied in this role with features such as late potentials on signal averaged ECG, QRS duration and QT prolongation studied with no reliable predictive capability for these measures except in select groups. ${ }^{8}$ QRS fragmentation on ECG is thought to represent wave fronts of heterogeneous conduction. In a retrospective trial of 998 patients, Das et at demonstrated that fragmented QRS (fQRS) on ECG was predictive of cardiac events (myocardial infarction (MI), need for revascularisation or cardiac death), but not for all-cause mortality. A retrospective trial of 10904 Finnish subjects suggested that $\mathrm{fQRS}$ was a relatively common finding and was only predictive of all-cause, cardiac or arrhythmic death if the subject had known cardiac disease. ${ }^{10}$

Magnetocardiography (MCG) is a non-invasive, noncontact body-surface method which uses magnetometers to measure and map the magnetic fields generated by the electrical activity within the heart. Compared with the ECG it offers the potential of superior spatial resolution, better detection of currents tangential to the body surface and less interference from non-cardiac structures. This may allow superiority in detecting conduction characteristics consistent with arrhythmogenesis. ${ }^{11}$ Korhonen $e t$ $a l^{12}$ studied 100 patients with remote MI presenting with or without ventricular tachycardia (VT) comparing late fields on signal averaged ECG and MCG. Late fields in the MCG QRS were more sensitive and equally specific in the VT group, even in patients with severe left ventricular systolic dysfunction where late fields on signal averaged ECG lost its discriminative ability. ${ }^{12}$ In a prospective trial of 158 patients (in sinus rhythm and atrial fibrillation) with at least mildly impaired left ventricular systolic function (left ventricular ejection fraction $(\mathrm{LVEF})<50 \%$ ) and recent MI, an MCG was recorded and analysed for fragmentation of the MCG QRS complex. ${ }^{13}$ Participants were followed up for an average of 50 months using hospital admission data to evaluate the endpoint of death. Arrhythmic death was ascertained by using available hospital admission data. This study demonstrated that while QRS duration on surface ECG could predict allcause death, QRS fragmentation on MCG was also able to predict arrhythmic death. Repolarisation abnormalities associated with VA have been studied in MCG. A trial of 49 patients with dilated cardiomyopathy presenting with or without VA underwent MCG recording; prolongation of the end part of the MCG T-wave was associated with the VA group. ${ }^{14}$ Kawakami et $a l^{15}$ prospectively studied VA risk in 51 patients with non-ischaemic cardiomyopathy for a mean follow-up of 2.9 years. They found that left intraventricular disorganised conduction (described as a deviation from the typical global clockwise left ventricle depolarisation) on MCG was predictive of major adverse cardiac events. ${ }^{15}$

Although MCG has shown promise in the role of detecting arrhythmic substrate, its application has been limited by the availability of the technology. Established MCG systems use superconducting quantum interference device (SQUID) sensors operating at around $77 \mathrm{~K}$ and largely require shielded environments to achieve an MCG recording that is discernible from background interference. This requires a significant cost of installation and operation that has prevented the technology being applied in mainstream medical practice. The Vitalscan device (Creavo Medical Technologies, Coventry, UK) is a new generation MCG device where the magnetometers operate at room temperature and has been designed to be mobile, operate in unshielded environments and require minimal training to perform. The aim in its design was to make MCG a more accessible and feasible technology in cardiology diagnostics.

The MAGNETO-SCD trial has been designed to evaluate MCG features that may be predictive of VA risk using the Vitalscan device in a group of patients undergoing ICD implantation for both primary and secondary prevention according to current national and international guidelines. $^{12}$

\section{STUDY DESIGN}

The MAGNETO-SCD trial is a prospective multicentre observation study. Patients undergoing first time implantation of an ICD or cardiac resynchronisation therapydefibrillator (CRT-D) implantation are being recruited. Prior to implantation of the device, they undergo a 15-minute MCG recording. All participants will receive the MCG recording, so there will be no randomisation. Participants will be followed up in the ICD clinic according to local protocol. The MCG analysis will be performed and correlated to ICD events including treatment of VA, detection of VA and burden of ventricular ectopy. Established MCG risk parameters such as QRS fragmentation will be identified and novel MCG parameters will be developed using unsupervised machine-learning algorithms. ${ }^{16}$ Statistical analysis identified a target sample size of 270 
patients (see below). This was based on an event rate of $23 \%$ at 20 months (based on both primary and secondary prevention ICD trials). ${ }^{5}$

The MAGNETO-SCD trial started recruitment in September 2019. There is a minimum follow-up of 4 years with a maximum follow-up of 6 years. Interim results are expected to be reported at the end of 2021 (minimum follow-up at 12 months).

\section{Objectives}

The primary objective is to identify and assess the precision and accuracy of MCG parameters in the prediction of future ICD therapies for VA in patients meeting criteria for ICD implantation under current guidelines. The primary endpoint is the delivery of appropriate therapy by the ICD device. Therapies will be reviewed by the principal investigators and Trial Steering Committee to evaluate whether they are considered appropriate

The secondary objectives are (a) to identify and assess the precision and accuracy of MCG parameters in the prediction of future VA in patients meeting the criteria for implantation under national and international guidelines, (b) to explore if the VitalScan MCG device is able to demonstrate similar findings to previous research using SQUID-based MCG technology, (c) to compare the predictive power of MCG to ECG parameters and LVEF for VA, (d) to explore the correlation between MCG parameters and LVEF, (e) to explore the effect of ICD therapy on quality of life, (f) whether MCG parameters can predict future inappropriate ICD therapies as they are known to increase mortality risk; if the study can identify patients that are not predicted to have SCD based on MCG parameters but are at increased risk of inappropriate ICD therapies, this may be a very important subgroup to avoid implanting an ICD.

\section{Participants}

The target population for this study is anyone listed for an ICD following an arrhythmia multidisciplinary team meeting who fulfil current national/international guidelines for new ICD or CRT defibrillator implantation $^{12}$ who do not have a device in situ already. This will include any aetiology of cardiomyopathy and both primary and secondary prevention indications. All trial visits and procedures will be conducted at University Hospital Coventry and Warwickshire (UHCW) National Health Service (NHS) Trust, UK and University Hospital of North Midlands NHS Trust, UK. We expect the participants will fall into two categories: (a) those at highest risk with a secondary prevention indication and (b) those at lower risk with a primary prevention indication \pm nonischaemic aetiology.

\section{Patient and public involvement}

The trial protocol and documentation were reviewed by the UHCW Patients and Public Involvement Group. Feedback from this group was taken into account in revising the Patient Information Sheet. Feedback regarding the

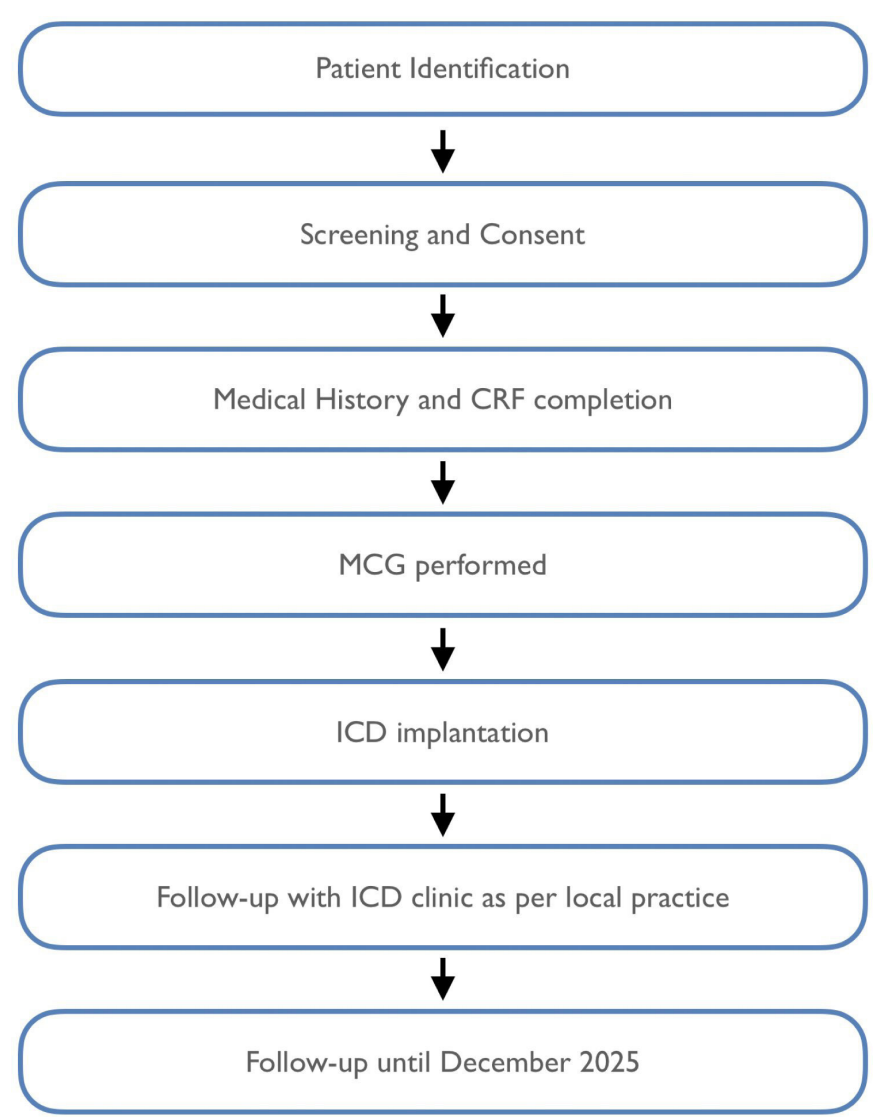

Figure 1 The MAGNETO-SCD flow from recruitment to end. CRF, case report form; ICD, implantable cardioverter defibrillator; MCG, magnetocardiogram; SCD, sudden cardiac death.

trial design and protocol was obtained with no suggestions for modification.

\section{Inclusion criteria}

Patients must be 18 years or older and able to give written informed consent (consent form attached as online supplemental file) to be included in the trial. Patients must meet national/international guidance for ICD implantation either for primary or secondary prevention of any aetiology.

\section{Exclusion criteria}

Patients are excluded from this trial if they are unable to lie still on a bed at a maximum of $30^{\circ}$ upright angle for $15 \mathrm{~min}$, if they have ongoing MI or active ischaemia (as evidenced by ECG changes, symptoms of chest pain or circulating cardiac biomarkers) or if they are deemed clinically unstable by their attending clinician. Patients with existing thoracic metallic implants (ie, pacing or defibrillator devices, metallic heart valves) will be excluded from the trial due to magnetic interference on the MCG device.

\section{Study plan}

The trial flow chart is shown in figure 1 and minimum follow-up is 4.5 years. 


\section{Screening and eligibility assessment}

Patients who have been identified as needing an ICD will be considered for participation in the trial and will be drawn from both inpatient and outpatient groups. Once identified the research team evaluate their suitability against inclusion and exclusion criteria. If the patient is eligible, the research team will inform the responsible clinical team and approach the patient either in person or via a telephone consultation.

\section{Baseline assessment}

Once recruited demographic information, medical history, current medication and imaging of LVEF (either by echocardiography, MRI or nuclear medical imaging) is documented along with resting 12-lead ECG. Participants then undergo an MCG recording using a VitalScan MCG device in an unshielded location that has been previously identified as having a favourable level of background electromagnetic interference. The MCG is recorded on an MRI safe bed of aluminium construction to reduce the impact of bed vibration on the magnetic field under the scanner head. Scans are recorded with the participant lying horizontal with a maximum of $30^{\circ}$ head up tilt if the participant is unable to tolerate a totally flat bed. The centre of the hexagonal MCG array is aligned to the fifth rib, 3-7 cm to the left of the left sternal edge (approximately at the location of V3 on a 12-lead ECG lead location). The MCG will be taken for 15 min to provide a large sample for signal averaging and stored on the device. Periodically, MCG recordings will be archived from the VitalScan device to ensure data integrity and to ensure adequate storage space on the device to allow continued recording.

All implanted ICDs will be programmed with extended detection algorithms to avoid labelling arrhythmias destined to be non-sustained as those that are potentially requiring ICD therapy for prevention of sudden death in accordance with current data. ${ }^{17}$ We also plan to analyse whether the VA event detected \pm shocked is ventricular tachycardia or ventricular fibrillation based on ventricular rate and electrogram characteristics (rhythm regularity and morphology) following review by the trial management group. All patients will be optimised with regard to medical therapy in accordance to published guidelines. ${ }^{2}$

Baseline blood tests and circulating vascular biomarker (high-sensitivity troponin, NT-pro brain natriuretic peptide, $\mathrm{C}$ reactive protein (CRP), high-sensitivity CRP, interleukin-6) will be collected and correlations made with primary and secondary endpoints.

\section{Follow-up assessment}

Participants will undergo standard care follow-up through the ICD clinic at their local centre (6 weeks post-implant and 6-12 monthly thereafter with remote monitoring based on patients' preference). Details of any arrhythmia or ventricular ectopic burden will be collected. Patient clinical management will be as per standard clinical care; no additional trial specific procedures will be performed.

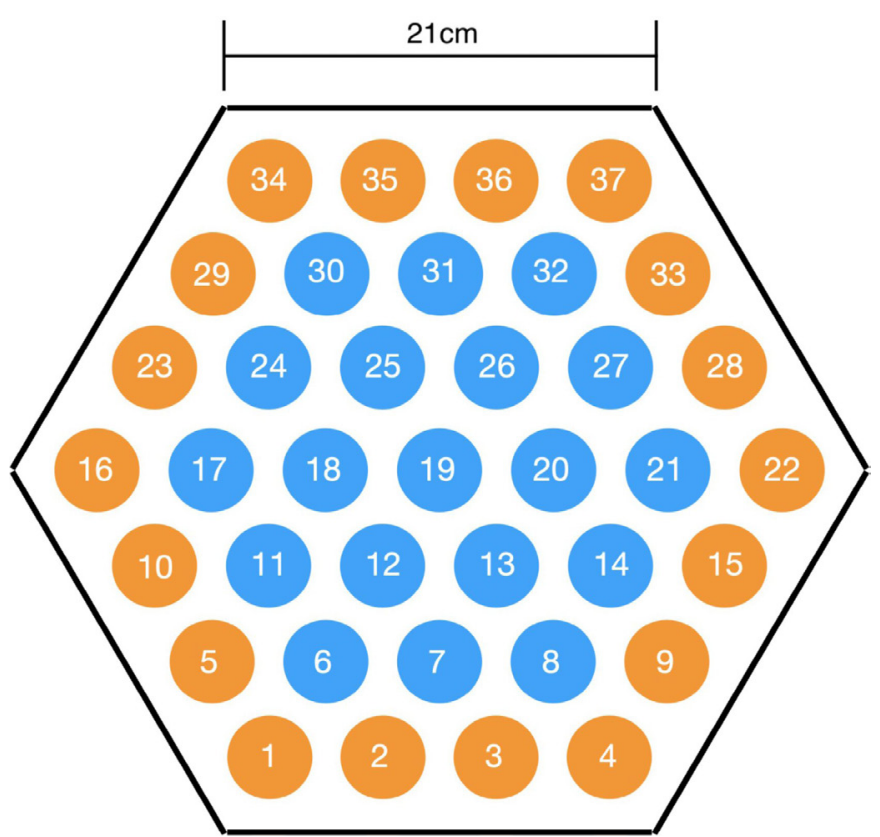

Figure 2 Scan head schematic showing sensor arrangement and locations.

At 12 months following trial commencement, the Trial Steering Committee (TSC) will evaluate the presence of endpoints and the timing of the first endpoint if present.

\section{MCG analysis}

MCGs from the VitalScan device are recorded at 2100 $\mathrm{Hz}$ in their raw form as a derivative of the magnetic field under a 37-array sensor head in a hexagonal orientation (figure 2). MCG recordings will be transferred to the core lab and analysed in custom software in a python programming environment. Recordings are filtered using a notch and moving average filter at $50 \mathrm{~Hz}$ to remove magnetic fields associated with mains electricity. Principal component analysis is used to identify point sources of interference where present and to subtract them from the final waveform for analysis. Once the signal has been appropriately prepared, it will be analysed for VA risk.

Fragmentation scoring will be calculated as described by Muller et al. ${ }^{18}$ After signal filtering, MCG channels are normalised and a noise level defined as the difference between the maximum and minimum signal in a 40 msec section occurring 150 msec after the R peak. Every signal extrema within a manually defined QRS interval that exceeds this noise floor is identified. The sum of the differences of amplitudes (y) of neighbouring extrema is calculated and added to the absolute values of the first and last extrema. This sum is multiplied by the total number of extrema $(\mathrm{M})$ to give the fragmentation score $(\mathrm{S})$.

$$
S=M\left(\left|y\left(t_{1}\right)\right|+\left|y\left(t_{M}\right)\right|+\sum_{(k=2)}^{M}\left|y\left(t_{k}\right)\right|-\left|y\left(t_{k+1}\right)\right|\right)
$$


This dimensionless number quantifies fragmentation and is calculated for all channels of the MCG. These can be averaged across multiple channels or the whole sensor array to give a total fragmentation score. In a prospective trial of patients with ischaemic cardiomyopathy, a fragmentation score from seven central coaxial channels of a 67-channel MCG demonstrated a sensitivity of $23 \%$ and specificity of $96 \%$. This was superior to QRS duration on ECG (used currently in risk assessment) ${ }^{1}$ and when combined with LVEF below $30 \%$, sensitivity increased to $50 \% .^{13}$

QT spatial dispersion will be analysed as described by Van Leeuwen et al. ${ }^{19} \mathrm{Q}$ wave onset and $\mathrm{T}$ wave end are visually identified in each MCG channel to the nearest millisecond by cursor placement. The $\mathrm{T}$ wave in the acquired signals can often be bipolar in nature and may not return to baseline until shortly before the subsequent $\mathrm{P}$ wave; $\mathrm{T}$ wave end is defined as the time of the visually determined vertex (maximum curvature) of the signal following the inflection point after $\mathrm{T}$ apex. In biphasic signals the $\mathrm{T}$ apex is interpolated from the neighbouring channels. If the difference between the results of the two observers is $>5 \mathrm{msec}$ for the MCG and $10 \mathrm{msec}$ for the ECG, the values are reexamined and corrected, otherwise the results are averaged. Spatial dispersion is then quantified using a smoothness index (SI). SI quantifies local temporal differences and increases with greater variations in QT interval duration between neighbouring sensor locations. ${ }^{20}$ Variance from normal QT spatial dispersion was calculated by performing MCG on a group of normal patients and using these as weighting for correcting factors in the observed SI to produce a normalised SI (SIn). This was done to take into account the inhomogeneity seen in normal cardiac activity. In this retrospective trial, spatial MCG QT dispersion was superior to temporal QT dispersion in either ECG or MCG at discriminating between VT and non-VT groups. ${ }^{19}$ SIn produced the best discrimination between VT and non-VT groups. With an SIn cut-off set at 4.84 for the comparison of the VT and non-VT groups, SIn was able to obtain a sensitivity of $100 \%$ and a specificity of $77 \%$.

Late QRS activity will be calculated as described by Korhönen et al. ${ }^{12}$ After signal processing and filtering, MCG signals will be manually examined to identify QRS onset and offset by cursor placement. Signals are accepted conditional on a residual noise below $35 \mathrm{ft}$ in a window of 40 msec occurring 150 msec after $\mathrm{R}$ peak. QRS duration, root mean square amplitude of the magnetic field in the last $40 \mathrm{msec}$ of the QRS complex $\left(\mathrm{RMS}_{40}\right)$, and duration of the low amplitude signal below $300 \mathrm{ft}\left(\mathrm{LAS}_{300}\right)$ in milliseconds. In this retrospective study of patients presenting with VT or no VT post-MI, prolonged $\mathrm{LAS}_{300}$ was most associated with the VT group with a relative risk of 1.08 (1.02-1.13). ${ }^{12}$ All MCG analysis will be performed by researchers blinded to patient outcomes. A Gantt chart detailing trial procedures is shown in table 1 .

The sensitivity and specificity of MCG parameters will be evaluated on the entire study cohort as well as assessing each parameter in groups defined by aetiology (ischaemic, non-ischaemic, cardiac channelopathy) and ICD implant indication (primary vs secondary). These

Table 1 Gantt chart detailing trial procedures

\begin{tabular}{|c|c|c|c|c|c|c|c|c|c|}
\hline \multirow[b]{2}{*}{ Procedure } & \multicolumn{5}{|l|}{ Phase 1} & \multicolumn{4}{|c|}{ Phase 2 (long-term follow-up) } \\
\hline & Screening & Baseline & $\begin{array}{l}6 \\
\text { weeks }\end{array}$ & $\begin{array}{l}3 \text { months } \\
\text { and every } \\
6 \text { months }\end{array}$ & $\begin{array}{l}\text { 1st } \\
\text { year }\end{array}$ & $\begin{array}{l}\text { 2nd } \\
\text { year }\end{array}$ & $\begin{array}{l}\text { 3rd } \\
\text { year }\end{array}$ & $\begin{array}{l}\text { 4th } \\
\text { year }\end{array}$ & $\begin{array}{l}\text { 5th } \\
\text { year }\end{array}$ \\
\hline Eligibility assessment & $x$ & $x$ & & & & & & & \\
\hline Informed consent & & $\mathrm{x}$ & & & & & & & \\
\hline $\begin{array}{l}\text { Demographic data (DOB, sex, ethnicity, height and } \\
\text { weight) }\end{array}$ & & $x$ & & & & & & & \\
\hline $\begin{array}{l}\text { Relevant clinical history-especially determining } \\
\text { arrhythmia aetiology }\end{array}$ & & $x$ & & & & & & & \\
\hline $\begin{array}{l}\text { Evaluation of left ventricular systolic function via } \\
\text { echocardiography, CMRI or radionucleotide imaging }\end{array}$ & & $x$ & & & & & & & \\
\hline Record current medications & & $x$ & & & & & & & \\
\hline Magnetocardiogram & & $x$ & & & & & & & \\
\hline ICD implantation & & $x$ & & & & & & & \\
\hline Routine ICD interrogation & & & $X$ & $X$ & $X$ & $X$ & $X$ & $X$ & $X$ \\
\hline
\end{tabular}

BNP, brain natriuretic peptide; BP, blood pressure; CMRI, cardiac MRI; CRP, C reactive protein; DOB, date of birth; GFR, glomerular filtration rate; hbA1c, haemoglobin A1c; ICD, implantable cardioverter defibrillator. 
groups are known to have different risks for future VA events. Data on late gadolinium enhancement from cardiac MRI will also be used to correlate MCG parameters and VA outcomes as this has recently been shown to be an important predictor of risk. ${ }^{21}$ We plan to categorise and evaluate MCG parameters in our cohort according to LVEF as this is a known risk predictor for VA events.

\section{Statistical analysis}

A total sample size of 270 participants was calculated for the study. Sample size adequacy was determined by ensuring precise estimates (small margin of error (ME)) for the diagnostic measure sensitivity. We aim to have a tool with at least $80 \%$ sensitivity and an ME of 5\%. Assuming that the study observed sensitivity will be $80 \%$, the sample size required to give a $10 \% \mathrm{ME}$ (corresponding to $95 \%$ CI, sensitivity being $70 \%-90 \%$ ) is 62 patients with the primary outcome of a VA event. Assuming that the prevalence of VA is $23 \%$ at 20-month follow-up in the overall study population, we will recruit 270 patients. Sensitivity, rather than specificity, was used for sample size determination since the prevalence of VA is less than $50 \%$ and hence smaller sample size for sensitivity than specificity calculations. This event rate has been calculated from existing literature using both primary and secondary prevention groups. ${ }^{5}$

$n=\left(\frac{1.96}{\text { Margin of Error }}\right)^{2} *$ Sensitivity $*(1-$ Sensitivity $) *\left(\frac{100}{\text { Event Rate }}\right)$

$$
n=\left(\frac{1.96}{0.1}\right)^{2} * 0.8 *(1-0.8) *\left(\frac{100}{23}\right)=267
$$

This will be combined with the observed prevalence in the cohort to calculate positive and negative predictive values. For the purpose of this trial, a negative predictive value is of greater clinical value and by powering the trial for sensitivity we are targeting a negative predictive value in excess of $90 \%$.

\section{Ethics and monitoring}

The trial design and research protocol were approved by the Yorkshire and Humber Sheffield Research Ethics Committee UK (Ref: 19/YH/0143) and Health Research Authority (IRAS reference 254466, EDGE ID: 123146) with informed consent being obtained from participants. The trial is being conducted in accordance with UK laws, Good Clinical Practice and the Declaration of Helsinki 2002. The MAGNETO-SCD TSC consists of independent specialists in arrhythmia management and a member of the Patients and Public Engagement Committee. The TSC are responsible for the conduct of the study and publication of results. They will meet annually to evaluate recruitment against targets, endpoints and any adverse or serious adverse device events.

\section{DISCUSSION}

Risk stratification for SCD and indications for ICD implantation are currently driven largely by LVEF assessment. There is a growing body of evidence that this strategy is in need of refinement. There are a group of patients who, based on current recommendations, do not qualify for an ICD, yet will go on to have an SCD event. Conversely, there are patients who qualify for an ICD, who never require them, exposing them to unnecessary morbidity and associated costs to either patients, healthcare providers or both. Where LVEF alone is used to evaluate SCD risk, the annual rate of ICD therapy can range from $1.1 \%$ to $5.1 \%{ }^{22}{ }^{23}$ The search for a safe but effective non-invasive risk stratification tool may be supported by detecting heterogeneous ventricular conduction and subsequent VA risk using MCG devices. However, the widespread adoption and depth of research into this technology has to date been hindered by the unavailability of MCG devices due to its prohibitive costs and complex installation requirements. The VitalScan MCG device represents one of a new generation of MCG recording machines designed for use in an unshielded clinical environment within room temperature to enable this largest prospective trial evaluating SCD risk using MCG in ICD recipients. This trial will evaluate previously identified MCG depolarisation characteristics associated with SCD risk using this novel MCG technology. The findings from this study may help identify those patients who qualify for an ICD by current guidelines, who never actually use their device and therefore are low risk. However, further studies will be needed to study MCG predictors of SCD in the general population, who currently do not qualify for an ICD.

The MAGNETO-SCD trial is required to help build an evidence base for the role of viable MCG devices in risk stratification of patients under consideration of ICD implantation. If this trial confirms a predictive role for MCG in this patient group, it would be logical to base future studies around different patient groups currently viewed as low risk.

\section{ETHICS AND DISSEMINATION}

The trial will be performed in accordance with the Declaration of Helsinki 2002 and conducted in accordance with the Research Governance Framework for Health and Social Care, the applicable UK Statutory Requirements (such as Medicines and Healthcare products Regulatory Agency) and Good Clinical Practice E6(R2); and carried out under a Clinical Trial Authorisation in accordance with the Medicines for Human Use Clinical Trials regulations. The protocol and subsequent amendments have been submitted to and approved by Research Ethics Committee prior to circulation. Personal data recorded on all documents will be regarded as strictly confidential and will be handled and stored in accordance with the General Data Protection Regulation 2016/679 and the Data Protection Act (2018). The Yorkshire and Humber Sheffield Research Ethics Committee UK (Ref: 19/ 
$\mathrm{YH} / 0143)$ and Health Research Authority (IRAS reference 254466, EDGE ID: 123146) have approved the current study.

All trial findings will be published in peer-reviewed journals and disseminated at appropriate conferences, departmental and scientific meetings.

\author{
Author affiliations \\ ${ }^{1}$ Cardiology, University Hospitals Coventry and Warwickshire NHS Trust, Coventry, \\ UK \\ ${ }^{2}$ Warwick Medical School, University of Warwick, Coventry, UK \\ ${ }^{3}$ Cardiology, Oxford University Hospitals NHS Foundation Trust, Oxford, UK \\ ${ }^{4}$ Cardiology, University Hospital of North Staffordshire NHS Trust, Stoke-on-Trent, UK
}

Correction notice This article has been corrected since it was first published. The middle name for Jamal Nasir Khan has been added.

Acknowledgements We would like to thank the Research and Development Department at UHCW NHS Trust for their support, Dr Peter Kimani (Statistician, University of Warwick) for statistical input and the UHCW PPIG for their input.

Contributors TL, HH, KS and FO contributed to the grant proposal, writing the protocol and are all involved in aspects of the day-to-day running of the trial. F0 is the chief investigator. He was involved in the conception of the trial and contributed to trial design. KS is the research trial manager. JK, KR, AP, AM-D and HR have been involved in trial design and protocol development and AM-D/AP are $\mathrm{Cl}$ at the Stoke site. TL and $\mathrm{HH}$ are responsible for trial recruitment and conduct of trial visits and undertaking MCG analyses.

Funding Creavo Medical Technologies have provided a research grant for this study but have no involvement in its running.

Competing interests None declared.

Patient consent for publication Not required.

Provenance and peer review Not commissioned; externally peer reviewed.

Supplemental material This content has been supplied by the author(s). It has not been vetted by BMJ Publishing Group Limited (BMJ) and may not have been peer-reviewed. Any opinions or recommendations discussed are solely those of the author(s) and are not endorsed by BMJ. BMJ disclaims all liability and responsibility arising from any reliance placed on the content. Where the content includes any translated material, BMJ does not warrant the accuracy and reliability of the translations (including but not limited to local regulations, clinical guidelines, terminology, drug names and drug dosages), and is not responsible for any error and/or omissions arising from translation and adaptation or otherwise.

Open access This is an open access article distributed in accordance with the Creative Commons Attribution Non Commercial (CC BY-NC 4.0) license, which permits others to distribute, remix, adapt, build upon this work non-commercially, and license their derivative works on different terms, provided the original work is properly cited, appropriate credit is given, any changes made indicated, and the use is non-commercial. See: http://creativecommons.org/licenses/by-nc/4.0/.

ORCID iD

Faizel Osman http://orcid.org/0000-0002-3962-5118

\section{REFERENCES}

1 NICE. Implantable cardioverter defibrillators and cardiac resynchronisation therapy for arrhythmias and heart failure. Technology Appraisal Guidance [TA314] Adler A, Appraisal Committee members, editors. 2014 Jun 25:1-68. Available: https:// www.nice.org.uk/guidance/ta314

2 pp. Priori SG, Blomström-Lundqvist C, Mazzanti A, et al. Esc guidelines for the management of patients with ventricular arrhythmias and the prevention of sudden cardiac death: the task force for the management of patients with ventricular arrhythmias and the prevention of sudden cardiac death of the European Society of cardiology (ESC). endorsed by: association for European paediatric and congenital cardiology (AEPC). vol. 36. Europ Heart $J$ 2015;2015:2793-867.

3 Moss AJ, Hall WJ, Cannom DS, et al. Improved survival with an implanted defibrillator in patients with coronary disease at high risk for ventricular arrhythmia. multicenter automatic defibrillator implantation trial Investigators. N Engl J Med 1996;335:1933-40.

4 Connolly SJ, Gent M, Roberts RS, et al. Canadian implantable defibrillator study (CIDS) : a randomized trial of the implantable cardioverter defibrillator against amiodarone. Circulation 2000;101:1297-302.

5 Zareba W, Cannom DS, Daubert JP, et al. Multicenter automatic defibrillator implantation trial II Investigators. prophylactic implantation of a defibrillator in patients with myocardial infarction and reduced ejection fraction. N Engl J Med 2002;346:877-83.

6 Kadish A, Dyer A, Daubert JP, et al. Prophylactic defibrillator implantation in patients with nonischemic dilated cardiomyopathy. $N$ Engl J Med 2004;350:2151-8.

7 Koplan BA, Stevenson WG. Ventricular tachycardia and sudden cardiac death. Mayo Clin Proc 2009;84:289-97.

8 Abdelghani SA, Rosenthal TM, Morin DP. Surface electrocardiogram predictors of sudden cardiac arrest. Ochsner J 2016;16:280-9.

9 Das MK, Saha C, El Masry H, et al. Fragmented QRS on a 12-lead ECG: a predictor of mortality and cardiac events in patients with coronary artery disease. Heart Rhythm 2007;4:1385-92.

10 Terho HK, Tikkanen JT, Junttila JM, et al. Prevalence and prognostic significance of fragmented QRS complex in middle-aged subjects with and without clinical or electrocardiographic evidence of cardiac disease. Am J Cardiol 2014;114:141-7.

11 Tavarozzi I, Comani S, Del Gratta C, et al. Magnetocardiography: current status and perspectives. Part I: physical principles and instrumentation. Ital Heart J 2002;3:75-85.

12 Korhonen P, Montonen J, Mäkijärvi M, et al. Late fields of the magnetocardiographic QRS complex as indicators of propensity to sustained ventricular tachycardia after myocardial infarction. $J$ Cardiovasc Electrophysiol 2000;11:413-20.

13 Korhonen P, Husa T, Tierala I, et al. Increased intra-QRS fragmentation in magnetocardiography as a predictor of arrhythmic events and mortality in patients with cardiac dysfunction after myocardial infarction. J Cardiovasc Electrophysiol 2006;17:396-401.

14 Korhonen $\mathrm{P}$, Väänänen $\mathrm{H}$, Mäkijärvi $\mathrm{M}$, et al. Repolarization abnormalities detected by magnetocardiography in patients with dilated cardiomyopathy and ventricular arrhythmias. J Cardiovasc Electrophysiol 2001;12:772-7.

15 Kawakami S, Takaki H, Hashimoto S, et al. Utility of high-resolution Magnetocardiography to predict later cardiac events in nonischemic cardiomyopathy patients with normal QRS duration. Circulat $J$ 2017;81:44-51.

16 Chen JH, Asch SM. Machine learning and prediction in medicine - Beyond the peak of inflated expectations. N Engl J Med 2017;376:2507-9.

17 Moss AJ, Schuger C, Beck CA, et al. Reduction in inappropriate therapy and mortality through ICD programming. N Engl J Med 2012;367:2275-83.

18 Müller HP, Gödde P, Czerski K, et al. Magnetocardiographic analysis of the two-dimensional distribution of intra-QRS fractionated activation. Phys Med Biol 1999;44:105-20.

19 Van Leeuwen P, Hailer B, Lange S, et al. Spatial distribution of repolarization times in patients with coronary artery disease. Pacing Clin Electrophysiol 2003;26:1706-14.

20 Van Leeuwen P, Hailer B, Wehr M. Spatial distribution of QT intervals: an alternative approach to QT dispersion. Pacing Clin Electrophysiol 1996;19:1894-9.

21 Di Marco A, Anguera I, Schmitt M, et al. Late gadolinium enhancement and the risk for ventricular arrhythmias or sudden death in dilated cardiomyopathy. JACC: Heart Failure 2017;5:28-38.

22 Bardy GH, Lee KL, Mark DB, et al. Amiodarone or an implantable cardioverter-defibrillator for congestive heart failure. N Engl J Med 2005;352:225-37.

23 Sabbag A, Suleiman M, Laish-Farkash A, et al. Contemporary rates of appropriate shock therapy in patients who receive implantable device therapy in a real-world setting: from the Israeli ICD registry. Heart Rhythm 2015;12:2426-33. 